\title{
Schistosoma mansoni in a low-prevalence area in Brazil: the importance of additional methods for the diagnosis of hard-to-detect individual carriers by low-cost immunological assays
}

\author{
Rafaella Fortini Queiroz Grenfell', Watson Martins', Martin Enk1, Áureo Almeida1, \\ Liliane Siqueira', Vanessa Silva-Moraes', Edward Oliveira², \\ Nídia Francisca de Figueiredo Carneiro ${ }^{3}$, Paulo Marcos Zech Coelho ${ }^{1 /+}$ \\ ${ }^{1}$ Laboratório de Esquistossomose ${ }^{2}$ Laboratório de Pesquisas Clínicas, Centro de Pesquisas René Rachou-Fiocruz, Belo Horizonte, MG, Brasil \\ ${ }^{3}$ Centro de Controle de Zoonoses, Montes Claros, MG, Brasil
}

Schistosomiasis diagnosis is based on the detection of eggs in the faeces, which is laborious and lacks sensitivity, especially for patients with a low parasite burden. Immunological assays for specific antibody detection are available, but they usually demonstrate low sensitivity and/or specificity. In this study, two simple immunological assays were evaluated for the detection of soluble Schistosoma mansoni adult worm preparation (SWAP) and egg-specific IgGs. These studies have not yet been evaluated for patients with low parasite burdens. Residents of an endemic area in Brazil donated sera and faecal samples for our study. The patients were initially diagnosed by a rigorous Kato-Katz analysis of 18 thick smears from four different stool samples. The ELISA-SWAP was successful for human diagnosis with $90 \%$ sensitivity and specificity, confirming the Kato-Katz diagnosis with nearly perfect agreement, as seen by the Kappa index (0.85). Although the ELISA-soluble S. mansoni egg antigen was $85 \%$ sensitive, it exhibited low specificity (80\%; Kappa index: 0.75) and was more susceptible to cross-reactivity. We believe that immunological assays should be used in conjunction with Kato-Katz analysis as a supplementary tool for the diagnosis of schistosomiasis for patients with low infection burdens, which are usually hard to detect.

Key words: Schistosoma mansoni - immunodiagnosis - low parasite burden - endemic area individuals - additional diagnosis

Schistosomiasis is endemic in many countries in the developing world and continues to be a serious public health threat. It is the most important of the human helminths in terms of morbidity and mortality and is associated with a subtle, but persistent morbidity (Utzinger et al. 2000). Despite major advances in its control, it is a chronic and debilitating disease with active transmission not only in highly endemic areas, but also in previously non-endemic areas (King \& Dangerfield-Cha 2008).

The estimation of the intensity of schistosomiasis is currently based on quantitative egg counts by the KatoKatz technique (Katz et al. 1972), as per the classification put forth by the World Health Organization (WHO 2002). Diagnosis that is solely based on the presence of ova by microscopic examination may lack sensitivity, especially for patients with light infections (Siqueira et al. 2011). Innovative and useful methods have been developed by our group, such as a saline gradient system for egg counts (Coelho et al. 2009), a miracidia hatching device for miracidia visualisation (Jurberg et al. 2008) and the DNA detection of eggs (Gomes et al. 2010). However, the sensitivity of parasitological methods decreases in

Financial support: CNPq-Decit 34/2008), FAPEMIG, FIOCRUZ, CPqRR, PPSUS

PMZC and RFQG are senior fellow and doctoral fellow, respectively, from CNPq.

+ Corresponding author: coelhopm@cpqrr.fiocruz.br

Received 25 September 2012

Accepted 7 January 2013 areas of low endemicity (WHO 2002). These methods do not allow for the detection of the infectious stage if the eggs are eliminated from the faeces prior to diagnosis.

Antibody detection assays can be highly specific and sensitive (Abdel-Fattah et al. 2011). These tests are promising for the diagnosis of patients living in lowendemicity areas, especially if used concurrently with coproscopy (Gonçalves et al. 2006, Igreja et al. 2007). The simultaneous use of multiple diagnostic methods has been applied to monitor the human population, to identify the small number of infected people once morbidity control is achieved with higher sensitivity and to diagnose early stages of the infection (WHO 2008, Coelho et al. 2009).

The main goal of this work was to properly standardise and evaluate ELISA using worm or egg antigens as alternatives to the previously studied serological tests. The performance of these assays was evaluated with sera from Brazilian individuals living in low-endemicity areas for Schistosoma mansoni infection. These results were then compared to 18 slides of faeces obtained on four different days diagnosed by the Kato-Katz method to estimate the true intensity of infection.

\section{SUBJECTS, MATERIALS AND METHODS}

Community survey - This study was performed in the communities of Buriti Seco and Morro Grande in Pedra Preta, a small village in an area endemic for schistosomiasis in the rural region of Montes Claros, state of Minas Gerais in the Southeast Region of Brazil (Siqueira et al. 2011). This area was chosen because the population had 
not been treated for schistosomiasis and it also had a low migration index with a fixed resident population. Additionally, according to data provided by Montes Claros Control Centre of Zoonosis, an infection rate of $12 \%$ was measured in 2005. Forty people from Pedra Preta aged 28-64 participated in this study (female/male: 22/18). Additionally, 20 healthy donors aged 22-65 participated as negative controls throughout the standardisation and evaluation of the assay (female/male adults: 14/06).

Sera and stool samples - Four stool samples and one serological sample were collected per individual on four consecutive days using $100 \mathrm{~mL}$ plastic tubes. The samples were identified using the name and number of the participant and, in cases of individuals living within the endemic area, the identification of the residence. Written informed consent was obtained from all of the participants. Eighteen glass slides $(41.7 \mathrm{mg} / \mathrm{smear})$ were evaluated for the presence of $S$. mansoni and other helminth eggs by the Kato-Katz technique (Katz et al. 1972) as follows: 12 slides of the first sample and two slides each of the second, third and fourth samples for a total of $750 \mathrm{mg}$ of faeces. The intensity of the infection was expressed as eggs per gram (epg) of faeces using the arithmetic mean of the egg counts obtained from the 18 slides multiplied by 24 .

Treatment of positive cases - All of the participants who were positive for schistosomiasis were treated with praziquantel in a single dose of $50 \mathrm{mg} / \mathrm{kg}$. Infections with other helminths were treated with a single dose of $400 \mathrm{mg}$ albendazole as recommended by the Brazilian Ministry of Health. The positive patients were resubmitted for stool examination by the Kato-Katz assay 30 days post-chemotherapy and were retreated as needed.

Preparation of antigens - Soluble S. mansoni adult worm antigen preparation (SWAP) - Swiss Webster female mice (4-6 weeks) were subcutaneously infected with 100 cercariae. After 45 days, the animals were sacrificed by cervical dislocation and underwent perfusion of the hepatic portal system using $0.85 \%$ saline solution plus $50 \mathrm{U} / \mathrm{L}$ heparin (Pellegrino \& Siqueira 1956). The adult worms were washed three times with $0.15 \mathrm{M}$ phosphate buffer saline (PBS), pH 7.2, subjected to mechanical grinding (Virtiz Precisa, Switzerland) and centrifuged at $9,500 \mathrm{~g}$ for $1 \mathrm{~h}$ at $4^{\circ} \mathrm{C}$ (Eppendorf AG, Germany). The supernatant obtained was dialysed in $0.9 \%$ saline solution in a cellulose membrane (Sigma-Aldrich, USA) for $48 \mathrm{~h}$ at $4^{\circ} \mathrm{C}$. The antigen was centrifuged at $1,250 \mathrm{~g}$ for $15 \mathrm{~min}$ at $4^{\circ} \mathrm{C}$ and the supernatant was stored at $-20^{\circ} \mathrm{C}$. An aliquot was submitted for protein quantification (Nanodrop, Thermo Scientific 2000, USA) and the obtained concentration was used as a parameter in the standardisation of the immunoassay for the detection of human and murine antibodies, herein known as the ELISA-SWAP.

Soluble S. mansoni egg antigen (SEA) - After performing the perfusion of the hepatic portal system of infected mice, the livers of these animals were removed for egg recovery. The antigen used in this study was prepared as previously described (Colley et al. 1978). The eggs of $S$. mansoni were homogenised and ground in
Virtiz (Virtiz Precisa) with $0.85 \%$ saline solution for 40 $\min$. The homogenate was centrifuged at 9,500 $g$ for $1 \mathrm{~h}$ at $4^{\circ} \mathrm{C}$. After $48 \mathrm{~h}$ of dialysis in $0.9 \%$ saline solution in a cellulose membrane (Sigma-Aldrich), the supernatant was submitted for protein assessment (Nanodrop, Thermo Scientific, USA). The final concentration was used as a parameter in the standardisation of the immunoassay for the detection of human and murine antibodies, herein known as the ELISA-SEA.

Evaluation of ELISA - The ELISA-SWAP and ELISA-SEA were first standardised with sequential incubations of diluted sera (1:50, 1:150, 1:300 in PBS), diluted conjugate $(1: 40,000,1: 60,000,1: 80,000,1: 100,000$ in PBS-T $0.05 \%$ ) and finally with 3,3,5,5-tetramethylbenzidine substrate solution (TMB: $\mathrm{H}_{2} \mathrm{O}_{2}$ ) (Invitrogen, USA) to determine the reactivity of sera and anti-human $\operatorname{IgG}$ conjugated to peroxidase (IgG-HRP) using a dilution curve. Two patient sera were selected for the construction of the standard curve.

ELISA-SWAP - MaxiSorpTM surface microtitre plates (NUNC, Denmark) were sensitised with $100 \mu \mathrm{L} /$ well of $1 \mu \mathrm{g} / \mathrm{mL}$ of SWAP diluted in $0.05 \mathrm{M}$ carbonatebicarbonate buffer, $\mathrm{pH} 9.6$, for $16 \mathrm{~h}$ at $4^{\circ} \mathrm{C}$. The plates were washed three times with $0.15 \mathrm{M} \mathrm{PBS}, \mathrm{pH} 7.2$, with $0.05 \%$ Tween 20 (LGC Biotecnologia, BR) (washing buffer) and the non-specific sites were blocked with $10 \%$ foetal bovine serum (FBS) in washing buffer at $37^{\circ} \mathrm{C}$ for $1 \mathrm{~h}$. After another round of washing, $100 \mu \mathrm{L}$ of sera diluted 1:50 in PBS was added in triplicate into each well and the plates were incubated at room temperature (RT) for $1 \mathrm{~h}$. The plates were then subjected to more washing and were incubated at RT for $1 \mathrm{~h}$ with IgG-HRP (Southern Biotech, USA) diluted 1:60,000 in washing buffer. The plates were washed again and $100 \mu \mathrm{L}$ of substrate $\mathrm{TMB} / \mathrm{H}_{2} \mathrm{O}_{2}$ was added to each well. The reaction was stopped after $20 \mathrm{~min}$ of incubation in the dark by the addition of $50 \mu \mathrm{L} /$ well of $2 \mathrm{~N}$ sulphuric acid. The results were obtained as absorbance values at $450 \mathrm{~nm}$ in a microplate reader (Bio-Rad Laboratories 3550, JA).

ELISA-SEA - MaxiSorpTM surface microtitre plates (NUNC, Denmark) were sensitised with $100 \mu \mathrm{L} /$ well of $3 \mu \mathrm{g} / \mathrm{mL}$ SEA antigen diluted in $0.05 \mathrm{M}$ carbonatebicarbonate buffer, $\mathrm{pH} 9.6$, for $16 \mathrm{~h}$ at $4^{\circ} \mathrm{C}$. The plates were washed three times with $0.15 \mathrm{M}$ PBS, $\mathrm{pH}$ 7.2, with washing buffer and the non-specific sites were blocked with $10 \%$ FBS in washing buffer at $37^{\circ} \mathrm{C}$ for $1 \mathrm{~h}$. After another round of washing, $100 \mu \mathrm{L}$ of sera diluted 1:150 in PBS was added in triplicate into each well and the plates were incubated at RT for $1 \mathrm{~h}$. The plates were then subjected to washing steps and were incubated at RT for $1 \mathrm{~h}$ with IgG-HRP (Southern Biotech) diluted 1:40,000 in washing buffer. The plates were washed and the results were obtained as described for the ELISA-SWAP.

Statistical analysis - The absorbance values were analysed with Minitab software by the KolmogorovSmirnov normality test. Normally distributed data were analysed by Student's $t$ test and non-normally distributed data were analysed by the Mann-Whitney $U$ test using $\mathrm{p}$ 
$<0.05$ as the significance level. The significance levels for percentages were determined by chi-square $\left(\chi^{2}\right)$ analysis ( $p<0.05$ as significant). The sensitivity, specificity, cut-off values, receiver operating characteristic (ROC) curves, likelihood ratios and positive predictive values were determined by Prism 5 software. The agreement between the methods was measured using the Cohen (1968) coefficient and analysed according to the Landis and Koch (1977) definition with ComKappa 2.0 software: $1.00-0.81$ nearly perfect, $0.80-0.61$ substantial, $0.60-0.41$ moderate, $0.40-0.21$ fair, $0.20-0$ slight, and $<0$ poor.

Ethics - This project was approved by the Ethical Research Committee of the René Rachou Research Institute-Fiocruz (03/2008) and the National Brazilian Ethical Board (784/2008, CONEP 14886). The study objectives were presented and explained to all participants and written consent was obtained through signature before admission to this study.

\section{RESULTS}

The ELISA-SWAP and ELISA-SEA standardisation was first performed to determine the units of reactivity of sera and IgG-HRP using a dilution curve. Selected patient samples exhibited high reactivities by both ELISA tests and high parasite burdens by the Kato-Katz. Fig. 1 depicts the titration patterns of each selected sera.

The human diagnostic characteristics of the ELISA methods were first determined separately to evaluate the sensitivity and specificity of both assays. For this purpose, faecal samples from 40 selected inhabitants from the low-endemic area were diagnosed by the Kato-Katz method, as per the WHO and were divided into negative and positive patients. All of the Kato-Katz-positive patients presented low parasite burdens (1-39 epg; $\mu=12 \pm$ 11), but two had 156 and $555 \mathrm{epg}$ of faeces. In addition, 20 negative samples obtained from non-endemic area individuals were used as negative controls.

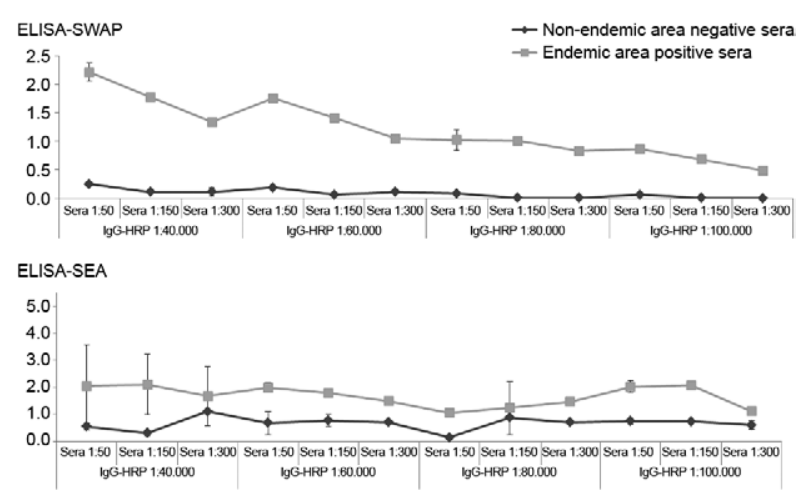

Fig. 1: human sera titration in ELISA-soluble Schistosoma mansoni adult worm antigen preparation (SWAP) and ELISA-soluble S. mansoni egg antigen (SEA). Antigen: SWAP or SEA $(1 \mu \mathrm{g} / \mathrm{mL}, 3 \mu \mathrm{g} / \mathrm{mL}$, respectively). Sera: endemic area positive sera and non-endemic area negative sera, diluted (1:50-1:300) in phosphate buffer saline (PBS). Conjugate: peroxidase labelled anti-human IgG conjugated to peroxidase (IgG-HRP) diluted (1:40.000-1:100.000) in PBS-Tween. Substrate: tetramethylbenzidine substrate solution.
The ELISA-SWAP was capable of detecting specific IgG antibodies in 18 of the 20 Kato-Katz-positive samples from patients from an endemic area for S. mansoni, including all but two samples with low parasite burdens (Fig. 2). In contrast, the ELISA-SEA detected 17 of the 20 Kato-Katz-positive patients (Fig. 3). The ELISASWAP also detected 18 of the 20 negative samples from the non-endemic residents, whereas the ELISA-SEA was able to diagnose 16 negative samples. The cut-off values of 0.15 for the ELISA-SWAP and 0.25 for the ELISASEA were determined by the ROC curve. A correlation was performed to verify the diagnostic concordance between the Kato-Katz egg counts and the diagnosis by both ELISA assays, as shown in Fig. 4.

Although the ELISA-SWAP and ELISA-SEA presented Kappa index correlations of $0.81( \pm 0.15)$ based on the Landis and Koch (1977) definition, the first method more strongly correlated with the Kato-Katz technique results, with a Kappa index of $0.85( \pm 0.16)$, indicating near perfect agreement. By comparison, the ELISA-SEA had a Kappa index of $0.75( \pm 0.16)$. Tables I, II show the comparison between each ELISA assay and the KatoKatz final diagnosis.

The analysis of discordant results revealed that 11 negative samples from endemic area patients were positive for IgG in both ELISAs. Furthermore, three KatoKatz-negative samples were positive by ELISA-SEA.

Because cross-reactivity can occur with indirect immunological assays, final analyses were performed for both ELISA techniques using sera samples from nine patients who were uniquely positive for Trichuris trichiura, hookworms, or Enterobius vermicularis. Among these nine patients, five exhibited high IgG titres by ELISASWAP and seven by ELISA-SEA (Table III).

Finally, the sensitivity values of the ELISA assays were determined by comparison of the absorbance values and the number of eggs estimated by the Kato-Katz technique. Importantly, only 14 patients showed eggs in the faeces when 12 slides of the first sample were analysed. Conversely, eggs were found in the faeces of more than six patients when 18 slides of four different samples were analysed. Therefore, using the complete analysis that diagnosed 20 patients as Kato-Katz-positive for schistosomiasis, the ELISA-SWAP was $90 \%$ sensitive and specific, whereas the ELISA-SEA was $85 \%$ sensitive and $80 \%$ specific.

\section{DISCUSSION}

The diagnosis of patients heavily infected with $S$. mansoni (high worm burden) can be easily performed with field-applicable parasitological methods (Gomes et al. 2010), including the urine-dipstick test used for the detection of schistosome cathodic circulating antigen. This dipstick has the potential to provide more sensitive and rapid detection of intestinal schistosomiasis in field-based surveys and it showed promising results in different parts of Africa (Ashton et al. 2011, Shane et al. 2011). However, it has become increasingly apparent that patients with low parasite burdens are unlikely to be correctly diagnosed. Mass treatment of individuals from endemic areas performed with single-dose oral treatment 


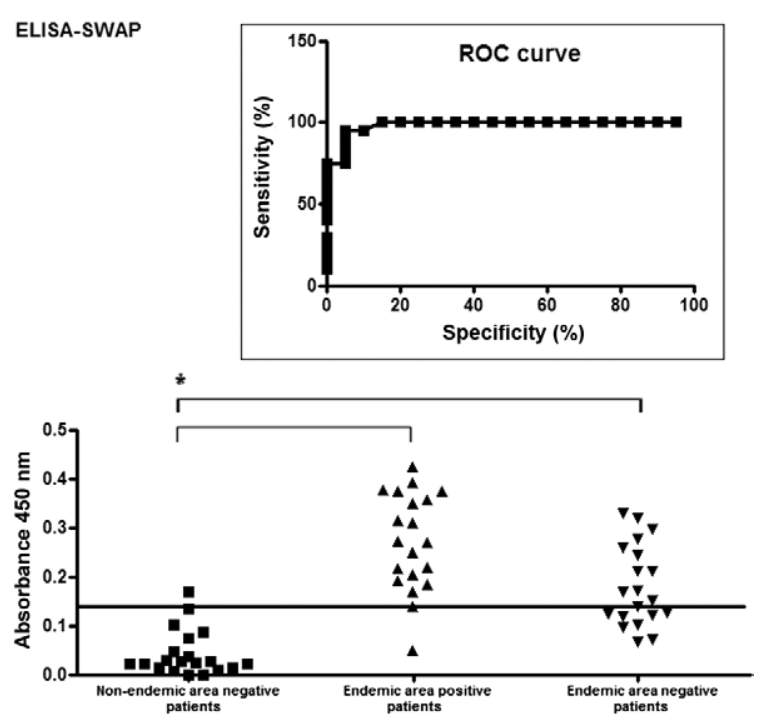

Fig. 2: reactivity of 60 serological samples from non-endemic and endemic individuals in ELISA-soluble Schistosoma mansoni adult worm antigen preparation (SWAP). Antigen: SWAP $(1 \mu \mathrm{g} / \mathrm{mL})$. Sera: nonendemic negative samples, endemic Kato-Katz positive and negative samples diluted 1:50 phosphate buffer saline-Tween (PBS-T). Conjugate: anti-human IgG conjugated to peroxidase (IgG-HRP) diluted 1:60,000 PBS-T. Substrate: tetramethylbenzidine substrate solution. Black line indicates the cut-off value of 0.15 . The box graphic indicates the receiver operating characteristic (ROC) curve for $90 \%$ of sensitivity and $95 \%$ of specificity. Statistical results are represented by an asterisk for $\mathrm{p}$ value $<0.001$

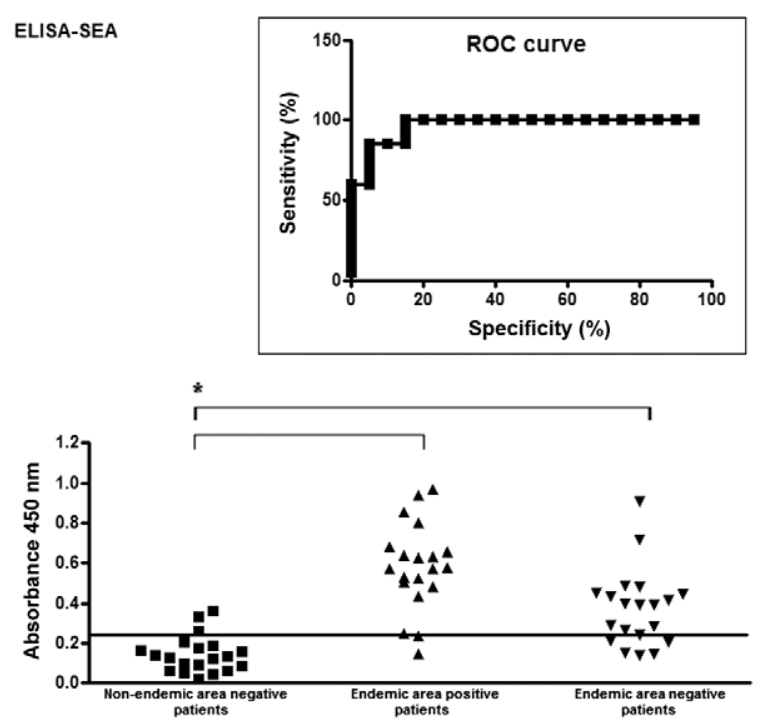

Fig. 3: reactivity of 60 serological samples from non-endemic and endemic individuals in ELISA-soluble Schistosoma mansoni egg antigen (SEA). Antigen: SEA (3 $\mu \mathrm{g} / \mathrm{mL})$. Sera: non-endemic negative samples, endemic Kato-Katz positive and negative samples diluted 1:150 phosphate buffer saline-Tween (PBS-T). Conjugate: anti-human $\operatorname{IgG}$ conjugated to peroxidase (IgG-HRP) diluted 1:40,000 PBS-T. Substrate: tetramethylbenzidine substrate solution. Black line indicates the cut-off value of 0.25 . The box graphic indicates the receiver operating characteristic (ROC) curve for $90 \%$ of sensitivity and $85 \%$ of specificity. Statistical results are represented by an asterisk for $\mathrm{p}$ value $<0.003$. can lead to persistence of low parasite burden infections. In an attempt to attain an accurate diagnosis for these patients, there is a need for sensitive diagnostic methods that can be used to confirm the results of parasitological methods that have poor sensitivity (Sorgho et al. 2005).

Patent schistosome infection is highly immunogenic and there is no difficulty in demonstrating the presence of anti-Schistosoma antibodies or cell-mediated immune responsiveness in infected subjects. Many different assays have been used to display such immunological reactivity, including skin hypersensitivity reactions against injected antigens, complement fixation, indirect immunofluorescence, indirect haemagglutination, radioimmunoassays and various flocculation and precipitation tests (Maddison 1991, Hamilton et al. 1998). Thus far, all of these methods have exhibited low sensitivities, demonstrating a lack of correlation between results from the direct and indirect methods. Furthermore, different antigens in immunological methods have been used to reduce cross-reactivity, such as microsomal adult worm antigen and circulating antigens (Maddison et al. 1985, de Jonge et al. 1990, Fillié et al. 1994). In this study, we evaluated the efficiency of two ELISA assays, based on the detection of $\operatorname{IgG}$ antibodies against easily obtained crude antigens (adult worm soluble antigens and egg soluble antigens), in reproducing the results obtained by an intensive search for positive cases by the Kato-Katz technique, which is considered by the WHO as the standard method for $S$. mansoni detection.

Worm antigens are an abundant and easily obtained source of antigenic material. Crude extracts of worms function well in ELISAs and generally provide higher sensitivity and specificity than those of larvae (Mott \& Dixon 1982). Antigens from schistosome eggs are also highly immunogenic, as their exit from the host depends on this immunogenicity. Consequently, anti-Schistosoma antibody titres rise after the onset of infection patency, as defined by the detection of eggs in clinical specimens (Doenhoff 1998). Notably, both types of antigens may lead to the development of low-cost diagnostic methods.

A detection method should be both sensitive and specific for human diagnosis. This applies not only to those living in endemic areas, but also to tourists and other travellers that may return home infected. In this study, 40 samples were obtained from individuals in an endemic area for $S$. mansoni and were examined by 18 glass slides using the Kato-Katz method. Of the 40 samples, 18 were from positive patients with a low parasite burden (1 to $200 \mathrm{epg} /$ faeces) and two were from positive patients with a high parasite burden (201-600 epg/faeces). The other 20 samples were negative for $S$. mansoni eggs by the KatoKatz technique. Additional 20 sera samples from nonendemic diagnosed individuals were obtained.

Due to the difficulty of diagnosing patients with low parasite burdens by the gold standard method, this study involved an intensive search for eggs in the faeces by the Kato-Katz method using 18 thick-smear slides from four different samples, a procedure that is not commonly performed in epidemiological surveillance studies. An important difference was noted when analysing 12 slides from a single sample, where only 14 patients were prop- 
erly diagnosed, while six patients were diagnosed when 18 slides were used. As observed by others, the absence of infection cannot be deduced from a reduced number of Kato-Katz thick smears and examination of multiple stool samples is recommended to more accurately estimate prevalence (de Vlas \& Gryseels 1992, Kongs et al. 2001, Booth et al. 2003).

We observed consistent results for the ELISA-SWAP in comparison to the Kato-Katz technique, reaching a Kappa index of 0.85 as a near-perfect agreement. Eighteen of the 20 negative non-endemic human samples were seen as negative by this immunological method. The same result was noted for the Kato-Katz-positive endemic area individuals, including 17 samples from patients with very low parasite burdens (1-40 epg/faeces) and one sample from a patient with a high parasite burden. Discordant results were found for 11 patients who were negative for $S$. mansoni eggs, but presented significant IgG titres. Not surprisingly, all of these patients were from endemic areas and could have been previously infected. Therefore, these data could represent cross-reactivity with other parasite antigens, could be secondary to previous infections or, although a large number of slides were analysed, they could represent the low sensitivity of the Kato-Katz technique for individuals with low parasite burdens. Nine of these individuals had eggs from other helminths in the faeces, such as hookworms, T. trichiura and E.vermicularis.

Finally, $90 \%$ of the Kato-Katz-positive cases and the non-endemic negative cases were properly detected by ELISA-SWAP. Based on the cut-off value of 0.150 , the sensitivity and specificity were calculated at $90 \%$. These data indicate that the methodology assumed in this work led to a higher sensitivity and specificity than immunological methods standardised by others (Nassr et al. 2002, Sorgho et al. 2005).

The analysis of the ELISA-SEA for non-endemic individuals indicated that 16 of the 20 sera samples were accurately diagnosed as negative. For the Kato-Katzpositive patients from endemic areas, three samples were diagnosed as false negatives without significant IgG titres. The Kappa index of 0.75 revealed a moderate agreement with the Kato-Katz technique as compared to the ELISA-SWAP (Kappa index of 0.81). The ELI-
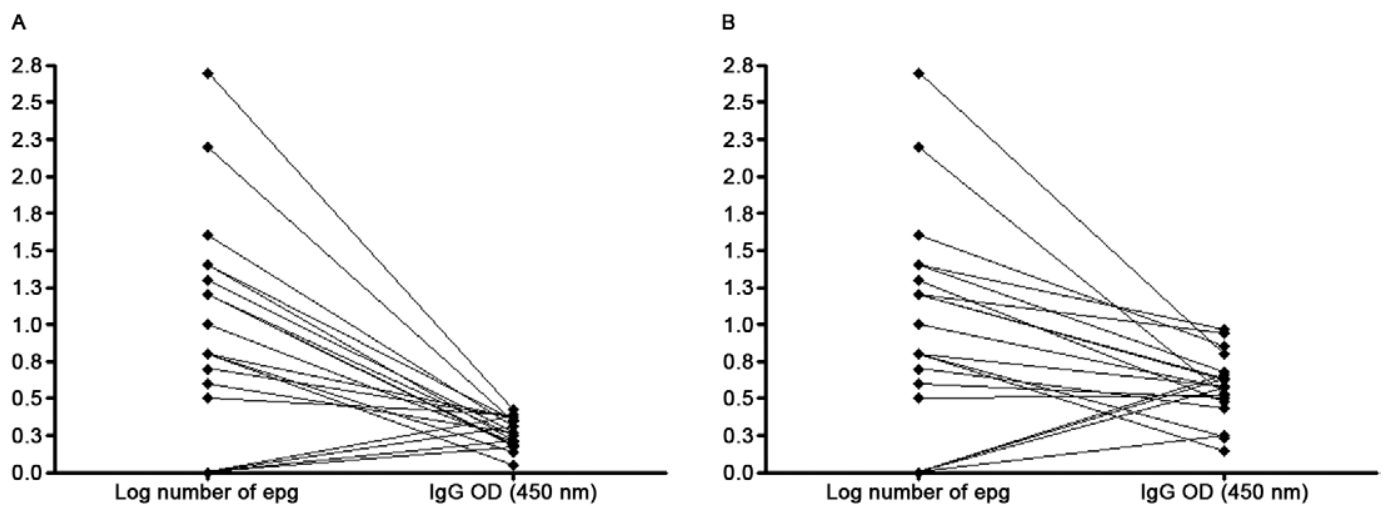

Fig. 4: correlation between $\operatorname{IgG}$ titres determined by ELISA-soluble Schistosoma mansoni adult worm antigen preparation (SWAP) (A) and ELISA-soluble S. mansoni egg antigen (SEA) (B) with the eggs count after 18 slides of Kato-Katz for endemic area positive patients. epg: eggs per gram of faeces; OD: optical density.

TABLE I

Comparison between ELISA-soluble Schistosoma mansoni adult worm antigen preparation (SWAP) with

Kato-Katz results obtained after the analysis of 18 slides from four faeces samples from endemic area positive patients

\begin{tabular}{lccc}
\hline & \multicolumn{2}{c}{ Kato-Katz results } & \\
\cline { 2 - 3 } ELISA-SWAP & $\begin{array}{c}\text { Positive } \\
\text { (n) }\end{array}$ & $\begin{array}{c}\text { Negative } \\
\text { (n) }\end{array}$ & $\begin{array}{c}\text { Total } \\
\text { (n) }\end{array}$ \\
\hline Positive & 19 & 0 & 19 \\
Negative & 1 & 20 & 21 \\
\hline Total & 20 & 20 & 40 \\
\hline
\end{tabular}

\section{TABLE II}

Comparison between ELISA-soluble Schistosoma mansoni egg antigen (SEA) with Kato-Katz results obtained after the analysis of 18 slides from four faeces samples from endemic area positive patients

\begin{tabular}{lccc}
\hline & \multicolumn{2}{c}{ Kato-Katz results } & \\
\cline { 2 - 3 } ELISA-SEA & $\begin{array}{c}\text { Positive } \\
(\mathrm{n})\end{array}$ & $\begin{array}{c}\text { Negative } \\
(\mathrm{n})\end{array}$ & $\begin{array}{c}\text { Total } \\
(\mathrm{n})\end{array}$ \\
\hline Positive & 19 & 3 & 22 \\
Negative & 1 & 17 & 18 \\
\hline Total & 20 & 20 & 40 \\
\hline
\end{tabular}


TABLE III

ELISA-soluble Schistosoma mansoni adult worm antigen preparation (SWAP) and ELISA-soluble $S$. mansoni egg antigen (SEA) results in individuals from endemic area with negative Kato-Katz results for S. mansoni, but positive for other helminthic infections (gray scale)

\begin{tabular}{|c|c|c|c|c|c|c|}
\hline \multirow[b]{2}{*}{$\begin{array}{l}\text { Endemic area } \\
\text { individuals }\end{array}$} & \multicolumn{6}{|c|}{ Positive $(+)$ or negative $(-)$ results } \\
\hline & Kato-Katz & ELISA-SWAP & ELISA-SEA & Hookworms & $\begin{array}{l}\text { Trichuris } \\
\text { trichiura }\end{array}$ & $\begin{array}{c}\text { Enterobius } \\
\text { vermicularis }\end{array}$ \\
\hline 1 & - & + & + & + & - & - \\
\hline 2 & - & - & + & + & - & - \\
\hline 3 & - & + & + & + & - & - \\
\hline 4 & - & - & + & + & - & - \\
\hline 5 & - & - & - & + & - & - \\
\hline 6 & - & + & + & + & - & - \\
\hline 7 & - & + & + & + & - & - \\
\hline 8 & - & + & - & + & - & + \\
\hline 9 & - & - & + & - & + & - \\
\hline
\end{tabular}

SA-SEA was less confident, with $80 \%$ specificity and $85 \%$ sensitivity for a cut-off of 0.250 . The ELISA-SEA data were very similar to data reported by other authors (Stothard et al. 2009) and they differed from the ELISASWAP data, which showed accurate confirmation of the Kato-Katz diagnoses.

Lack of sensitivity is a common problem for both parasitological and antigen/antibody-detection methods and immunological methods usually have poor specificity (i.e., a high proportion of egg-negative and antibody-positive results) (Doenhoff et al. 2004). Solutions are available, as we have shown. All of the data obtained in this work demonstrated that a simple technique, such as an ELISA using SWAP, can reach a suitable Kappa index agreement and can diagnose $S$. mansoni, even for individuals with very low egg outputs. As an additional tool, these indirect immunological methods can be used in association with a small number of slides for the Kato-Katz examination, as recommended by the $\mathrm{WHO}$, or with multiple slides and samples, as recommended by our work.

Results from individual laboratories and multicentre trials suggest that egg antigens provide greater diagnostic sensitivity and specificity than worm antigens for the detection of infection (McLaren et al. 1978, Mott \& Dixon 1982, Mott et al. 1987). In contrast, we have shown that the use of egg antigens for diagnosing schistosomiasis can be controversial due to false-positive results and cross-reactivity. The fact that there is an increase in anti-worm IgG after patency, in addition to anti-egg antibody titres, is perhaps best explained by the initial production of glycanic epitope-specific antibodies, which schistosome larvae and worms have in common (Alarcón et al. 2000). Extracts prepared by homogenising Schistosoma eggs contain a large number of molecules, although only a minority of the SEA constituents are released by viable eggs in vivo, as has been demonstrated in vitro (Ashton et al. 2001).
Briefly, the sensitivity and specificity of a single ELISA-SWAP examination has been confirmed. The ELISA-SEA presented consistently lower results than the ELISA-SWAP when compared to the Kato-Katz results and a significant number of false-positive cases when compared to the ELISA-SWAP. This warrants additional studies, especially for research directly related to patients with low schistosomiasis parasite burdens. Even though the Kato-Katz examination using 18 thick smears was rigorous, some infections might have been missed by this technique. Thus, we recommend further studies comparing ELISAs to more sensitive techniques than the Kato-Katz method. We have shown that two simple and well-known tests are indispensable for patient diagnosis and analysis for the rigorous monitoring of community-based helminth control programmes.

\section{REFERENCES}

Abdel-Fattah M, Al-Sherbiny M, Osman A, Charmy R, Tsang V 2011. Improving the detection limit of quantitative diagnosis of anti- $S$. haematobium antibodies using Falcon Assay Screening Test (FAST) ELISA by developing a new standard curve. Parasitol Res 108: 1457-1463.

Alarcón de Noya B, Colmenares C, Lanz H, Caracciolo MA, Losada S, Noya O 2000. Schistosoma mansoni: immunodiagnosis is improved by sodium metaperiodate which reduces cross-reactivity due to glycosylated epitopes of soluble egg antigen. Exp Parasitol 95: 106-112.

Ashton PD, Harrop R, Shah B, Wilson RA 2001. The schistosome egg: development and secretions. Parasitology 122: 329-338.

Ashton RA, Stewart BT, Petty N, Lado M, Finn T, Brooker S, Kolaczinski JH 2011. Accuracy of circulating cathodic antigen tests for rapid mapping of Schistosoma mansoni and S. haematobium infections in Southern Sudan. Trop Med Int Health 16: 1099-1103.

Booth M, Vounatsou P, N'goran EK, Tanner M, Utzinger J 2003. The influence of sampling effort and the performance of the Kato-Katz technique in diagnosing Schistosoma mansoni and hookworm coinfections in rural Côte d'Ivore. Parasitology 127: 525-531. 
Coelho PMZ, Jurberg AD, Oliveira AA, Katz N 2009. Use of a saline gradient for the diagnosis of schistosomiasis. Mem Inst Oswaldo Cruz 104: 720-723.

Cohen J 1968. Weighted kappa: nominal scale agreement with provisions for scales disagreement of partial credit. Psychol Bull 70: 213-220.

Colley DG, Lewis FA, Goodgame RW 1978. Immune responses during human schistosomiasis. IV. Induction of suppressor cell activity by schistosome antigen preparations and concanavalin A. J Immunol 120: 1225-1232.

de Jonge N, Kremsner PG, Krijger FW, Schommer G, Fillié YE, Kornelis D, van Zeyl RJ, van Dam GJ, Feldmeier H, Deelder AM 1990. Detection of the schistosome circulating cathodic antigen by enzyme immunoassay using biotinylated monoclonal antibodies. Trans R Soc Trop Med Hyg 84: 815-818.

de Vlas SJ, Gryseels B 1992. Underestimation of Schistosoma mansoni prevalences. Parasitol Today 8: 274-277.

Doenhoff MJ 1998. Granulomatous inflammation and the transmission of infection: schistosomiasis - and TB too? Immunol Today 19: 462-467.

Doenhoff MJ, Chiodini PL, Hamilton JV 2004. Specific and sensitive diagnosis of schistosome infection: can it be done with antibodies? Trends Parasitol 20: 35-39.

Fillié YE, Van Lieshout L, Kornelis D, Deelder AM 1994. Evaluation of an ELISA for combined measurement of CAA and CCA in schistosomiasis mansoni. Acta Trop 57: 279-287.

Gomes LI, Marques LHS, Enk MJ, Oliveira MC, Coelho PMZ, Rabello A 2010. Development and evaluation of a sensitive PCRELISA system for detection of Schistosoma infection in feces. PLoS Negl Trop Dis 4: e664.

Gonçalves MM, Barreto MG, Peralta RH, Gargioni C, Gonçalves T, Igreja RP, Soares MS, Peralta JM 2006. Immunoassays as an auxiliary tool for the serodiagnosis of Schistosoma mansoni infection in individuals with low intensity of egg elimination. Acta Trop 100: 24-30.

Hamilton JV, Klinkert M, Doenhoff MJ 1998. Diagnosis of schistosomiasis: antibody detection, with notes on parasitological and antigen detection methods. Parasitology 117 (Suppl.): S41-S57.

Igreja RP, Matos JA, Gonçalves MM, Barreto MM, Peralta JM 2007. Schistosoma mansoni-related morbidity in a low-prevalence area of Brazil: a comparison between egg excretors and seropositive non-excretors. Ann Trop Med Parasitol 101: 575-584.

Jurberg AD, de Oliveira AA, Lenzi HL, Coelho PMZ 2008. A new miracidia hatching device for diagnosing schistosomiasis. Mem Inst Oswaldo Cruz 103: 112-114.

Katz N, Chaves A, Pellegrino J 1972. A simple device for quantitative stool thick-smear technique in schistosomiasis mansoni. Rev Inst Med Trop Sao Paulo 14: 397-400.

King CH, Dangerfield-Cha M 2008. The unacknowledged impact of chronic schistosomiasis. Chronic Illn 4: 65-79.

Kongs A, Marks G, Verlé P, Van der Stuyft P 2001. The unreliability of the Kato-Katz technique limits its usefulness for evaluating $S$. mansoni infections. Trop Med Int Health 6: 163-169.
Landis JR, Koch GG 1977. The measurement of observer agreement for categorical data. Biometrics 33: 159-174.

Maddison SE 1991. Serodiagnosis of parasitic infections. Clin Microbiol 4: 457-469.

Maddison SE, Slemenda SB, Tsang VC, Pollard RA 1985. Serodiagnosis of Schistosoma mansoni with microsomal adult worm antigen in an enzyme-linked immunosorbent assay using a standard curve developed with a reference serum pool. Am J Trop Med Hyg 34: 484-494.

McLaren M, Draper CC, Roberts JM, Minter-Goedbloed E, Ligthart GS, Teesdale CH, Amin MA, Omer AH, Bartlett A, Voller A 1978. Studies on the enzyme linked immunosorbent assay (ELISA) test for Schistosoma mansoni infections. Ann Trop Med Parasitol 72: 243-253.

Mott KE, Dixon H 1982. Collaborative study on antigens for immunodiagnosis of schistosomiasis. Bull World Health Organ 60: 729-753.

Mott KE, Dixon H, Carter CE, Garcia E, Ishii A, Matsuda H, Mitchell G, Owhashi M, Tanaka H, Tsang VC 1987. Collaborative study on antigens for immunodiagnosis of Schistosoma japonicum infection. Bull World Health Organ 65: 233-244.

Nassr AKh, Hassan MM, Abdel Salam FM, Lashin AH, Shahin WA, Amin H 2002. IgG isotypes in schistosomiasis patients before and after praziquantel. $J$ Egypt Soc Parasitol 32: 931-952.

Pellegrino J, Siqueira A 1956. Técnica de perfusão para colheita de Schistosoma mansoni em cobaias experimentalmente infestadas. Rev Bras Malariol Doencas Trop 8: 589-597.

Shane HL, Verani JR, Abudho B, Montgomery SP, Blackstock AJ, Mwinzi PN, Butler SE, Karanja DM, Secor WE 2011. Evaluation of urine CCA assays for detection of Schistosoma mansoni infection in Western Kenya. PLoS Negl Trop Dis 5: e951.

Siqueira LMV, Coelho PMZ, de Oliveira AA, Massara CL, Carneiro NFF, Lima ACL, Enk MJ 2011. Evaluation of two coproscopic techniques for the diagnosis of schistosomiasis in a low-transmission area in the state of Minas Gerais, Brazil. Mem Inst Oswaldo Cruz 106: 844-850.

Sorgho H, Bahgat M, Poda JN, Song W, Kirsten C, Doenhoff MJ, Zongo I, Ouédraogo JB, Ruppel A 2005. Serodiagnosis of Schistosoma mansoni infections in an endemic area of Burkina Faso: performance of several immunological tests with different parasite antigens. Acta Trop 93: 169-180.

Stothard JR, Sousa-Figueiredo JC, Standley C, Van Dam GJ, Knopp S, Utzinger J, Ameri H, Khamis AN, Khamis IS, Deelder AM, Mohammed KA, Rollinson D 2009. An evaluation of urine-CCA strip test and fingerprick blood SEA-ELISA for detection of urinary schistosomiasis in schoolchildren in Zanzibar. Acta Trop 111: $64-70$.

Utzinger J, N'Goran EK, N'Dri A, Lengeler C, Xiao S, Tanner M 2000. Oral artemether for prevention of Schistosoma mansoni infection: randomized controlled trial. Lancet 355: 1320-1325.

WHO - World Health Organization 2002. Prevention and control of schistosomiasis and soil-transmitted helminthiasis. Tech Rep Ser 912: 1-63.

WHO - World Health Organization 2008. Report of the Scientific Working Group Meeting on Schistosomiasis, WHO-TDR-SWG, Geneva, 123 pp. 